\title{
A classification and framework for measuring sustainability supply chain risk indices in small and medium enterprises
}

Cite as: AIP Conference Proceedings 2097, 030001 (2019); https://doi.org/10.1063/1.5098176

Published Online: 23 April 2019

Agung Sutrisno, Vikas Kumar, Dwi Handayani, Rudi K. Arief, Shinta Virdhian, and Charles Punuhsingon

View Online

\section{Conference Proceedings}

Get $30 \%$ off all print proceedings!
Enter Promotion Code P/DF=30 at checkout

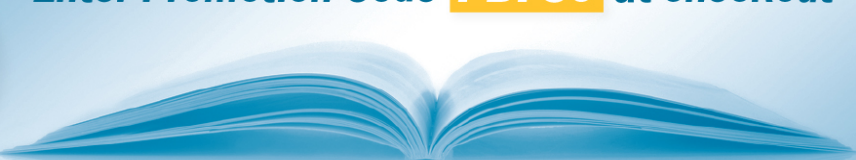




\title{
A Classification and Framework for Measuring Sustainability Supply Chain Risk Indices in Small and Medium Enterprises
}

\author{
Agung Sutrisno ${ }^{1, \mathrm{a})}$, Vikas Kumar, ${ }_{5, \mathrm{~b})}$, Dwi Handayani ${ }^{3, \mathrm{c})}$, Rudi K. Arief ${ }^{4, \mathrm{~d})}$, \\ Shinta Virdhian ${ }^{5, \mathrm{e})}$, Charles Punuhsingon ${ }^{6, \mathrm{f})}$
}

${ }^{I}$ Department of Mechanical Engineering, Sam Ratulangi University, Indonesia.

${ }^{2}$ Bristol Business School, the University of The West of England, United Kingdom.

${ }^{3}$ Department of Industrial Engineering, Universitas Islam Indonesia, Indonesia.

${ }^{4}$ Department of Mechanical Engineering, Muhammadiah University West Sumatera, Indonesia.

${ }^{5}$ Balai Besar Logam dan Mesin Bandung, Indonesia.

${ }^{6}$ Department of Mechanical Engineering, Sam Ratulangi University, Indonesia

a) Corresponding author: agungsutrisno@unsrat.ac.id.

b)Vikas.Kumar@uwe.edu.uk.

c)dwihandayani@uii.ac.id.

d) rudikarief@umsb.ac.id.

e) shinta_va@yahoo.com

f) charlespunuhsingon75@gmail.com

\begin{abstract}
Considering its significant contribution to the global Gross Domestic Product, improving an understanding of risks affecting the sustainability of operation in small and medium enterprises in a networked economy is undeniably important. Unfortunately, most of the previous studies concerning on managing risk in small and medium enterprises within a supply chain context are mostly focused on economic and operational risks and overlooking to the emerging risk, the sustainability risk. There is a need to have a better understanding of the mode of sustainability-risk variables affecting the operability of small and medium enterprises in supply chain context and framework for measuring their sustainability risk indices. This study, therefore, proposes a conceptual model to classify sustainability-related risk variables in the operation of the small and medium enterprises departing from the triple bottom lines namely, economic, environmental, and social risk from earlier references and develops a framework for measuring supply chain sustainability risk. proposed. The distinction between operational and sustainability risk variables and additional parameters for measuring the scale of risk score are presented. In the end, the paper provides new research directions for deeper investigation.
\end{abstract}

\section{INTRODUCTION}

With a growing contribution to the global GDP [1], improving sustainability in operations of small and medium enterprises in a networked economy against the adverse impact of business uncertainty is undeniably important. Nevertheless, owing to its limitation in business strategy expertise and resource scarcity to prevent adverse impact of uncertainty in their business operation [2], the rate of business failure of the SMEs is very high. Therefore, attention 
on identifying and mitigating the negative impact of risk factors affecting the sustainability of the Small and Medium Enterprises in their supply chain operational framework is important. In this regard, a study focusing on identifying, classifying and proposing a framework for appraising sustainability risk in the operation of SMEs within their supply chain is imperative with the consideration that capability to manage supply chain sustainability risk is one of the keys of competitive advantages [3]. However, earlier studies on managing risk in SMEs context as exemplified by scientific studies of $[4,5]$ are mostly focusing on common operational risk variables such production facility failure, demand mismatch and so on. Moreover, the establishment of risk taxonomy of the SMEs supply chain is important for risk treatment and management leading to effective risk mitigation [6]. The existence of studies intended for improving understanding of sustainability risk of the Supply Chain of SMEs is still scarce. Motivated by this scarcity, the aim of this paper is related to proposing a taxonomy of sustainability risk dimensions adopted from theoretical perspectives. The structure of this paper is as follows, the first overview of small and medium enterprises, risk management and sustainability risk are provided in section 2 . In section 3 , the classification between operational and sustainability risk dimensions in a supply chain is presented. In the end, conclusion and new opportunities for investigations are presented in section 4 .

\section{OVERVIEW OF SMALL AND MEDIUM ENTERPRISES, SUPPLY CHAIN RISK MANAGEMENT AND TAXONOMY OF SUPPLY CHAIN RISK}

In spite of its remarkable contribution to the global economy, there is no universally accepted definition of the SMEs. The definition of the SMEs varies from country to country [7], however, consensus on criteria of the SMEs is falling under same criterion such as, independently operated, having informal organizational structure, laborintensive, lack of resource to handle internal and external hazard, linked functions to personnel and doing research and development intuitively $[8,9,10]$.

Small and Medium Enterprises (SMEs) often face challenges linked to the resources available to tackle the impact of risk event occurrences. As a result, the operation of the small and medium enterprises is usually characterized by problematic situations such as human resource shortage, technological obsolescence, limited financial capital and lost risk capability absorbance [11]. Considering the above characteristics hindering the operation of their businesses, the rate of the mortality among SMEs is at large. Considering the adverse impact of above hindrance factors in running the SMEs business, appropriately identifying and managing on the risks faced by SMEs is becoming one of the prerequisites for reaping business success. Risk according to Qazi and Gaudensy [6] can be defined as the combination between two components, the likelihood of negative event occurrences and severity of their effects.

Supply chain risk can be defined as the negative impact of uncertainty at a micro and macro level in operations that affects the delivery of goods and service to their end customers [13]. A comprehensive definition by Yi and Stevenson [14] stated that supply chain risk management can defined as "the identification, assessment, treatment, and monitoring of supply chain risks, with the aid of the internal implementation of tools, techniques and strategies and of external coordination and collaboration with supply chain members so as to reduce vulnerability and ensure continuity coupled with profitability, leading to competitive advantage". Following Wang et al., [15], there are four general categories of the supply chain risks, logistics, information, customer and environmental risks.

As the unintended events negatively affect the operation of a supply chain, it is necessary to differentiate between operational and sustainable supply chain risk variables and identify appropriate ways to mitigate their adverse impacts. The operational risk variables usually cause temporary disruption to the supply chain meanwhile sustainability risk variables affect disruption in longer time horizon and hence if not managed properly may cause companies going to bankrupt. The supply chain risk management itself consists of risk identification, risk appraisal, risk evaluation, and risk mitigation.

The ability to identify and separate operational and sustainability risk variables enable decision-makers to determine appropriate corrective or preventative measures. Depending on its locus of occurrence, risk variables in a supply chain are divided into two categories, the internal and external supply chain risk. The internal supply chain risk is a kind of risk characterized by internal supply chain operation and it is usually easier to mitigate and the 
external supply chain risk is affected by the impact of uncertainty coming from outside of the supply chain operational environment. The modes of internal supply chain risks are in the form of information sharing risk, schedule, and product and process quality risk, faulty management decisions, financial problems, meanwhile the mode of external supply chain risk which is often called macro risk consist of economy-political risk, natural and man-made hazard, environmental and technological problems [16].

\section{Operational Supply Chain Risk}

The first category of supply chain risk taxonomy is operational risk variable. As the name implies, it is a kind of supply chain risk caused by operational disturbances due to operational related drivers disrupting supply chain operation. Some typical modes of operational supply chain risks are malfunction of production and distribution facility, erroneous in estimating supply and demand and lack of inventory etc. [18]. The adverse impact of operational supply chain risks is relatively short in terms of time duration consequences and easier to control since the locus of occurrence is still inside of the company. TABLE 1 collates some common operational supply chain risks identified from the literature.

TABLE 1. Typology of operational supply chain risk source

\begin{tabular}{lc}
\hline \multicolumn{1}{c}{ Risk Source } & References \\
\hline Supply Risk & Nalussamy and Ambedkar (2016) \\
Demand Risk & Sujka and Klarshik, 2012 \\
Production Risk & Nalussamy and Amdebkar (2016) \\
Delivery Risk & Gupta et al., (2014) \\
\hline
\end{tabular}

- Supply Risk: Supply risk is a kind of operational risk caused by inappropriate estimation in the amount of supply of goods and services to the end customers,

- Demand Risk: Demand risk is a typological operational risk caused by the wrong estimation in goods and service demand

- Production Risk: This third risk type concerns with negative uncertainty related to the disturbances in the production process caused by the defective product, worker absenteeism and manufacturing facility malfunction causing production process to halt

- Delivery Risk: Delivery risk is the negative uncertainty concerning on activities in delivering products and service $\mathrm{s}$ to the intermediate and end customers.

\section{Sustainability Supply Chain Risk}

In the last couple of decades, there is a pressure on supply chain operation to pay more attention to environmental and social aspect in delivering product and services to the end customers. This has led to the new emergent of a new concept known as supply chain risk-sustainability [19]. Different from operational risk, sustainability risks are driven by the occurrence of the adverse event causing negative environmental and social consequences at a large scale and continuation over a business operation in the longer time horizon. The distinct difference between operational and sustainability-related risk is that the latter brings adverse consequences to environmental and social relation [20]. Related to the time impact of the consequences, sustainability risk variables have long impact consequences and relatively harder to control compare to operational supply chain risk. The typical sustainability risk variables collected from various references are depicted in TABLE 2. 
TABLE 2. Typology of supply chain risk sustainability risk variables

\begin{tabular}{lc}
\hline \multicolumn{1}{c}{ Risk Dimension } & References \\
\hline Reputational Risk & Jesko et al., (2017), Petersen and Lemke (2015) \\
Competition Risk & Aon ( 2011) \\
Innovation Risk & Mansor et al., (2016) \\
Environmental Risk & Gianakis and Papadopoulos (2016) \\
Social Risk & Gianakis and Papadopoulos (2016), Davarzani et al., (2015), \\
& Rathore et al., (2017) \\
Regulatory Risk & Gianakis and Papadopoulos (2016) \\
Human Resources Risk & Meyer et al., (2011) \\
Security Risk & Zuo and Hu (2009) \\
Political Risk & Hadiguna (2017) \\
Cultural Risk & Galli (2018), Ragunath and Devi (2018) \\
Collaborative Risk & Friday et al., (2018) \\
Corruption Risk & Monteiro et al., (2018) \\
\hline
\end{tabular}

- Reputational risk: Reputational risk is defined as any risk affecting the degrading reputation or fame of a company.

- Competition Risk: In doing business, companies competing for one another in reaping their business goal. Competition risk in this context refers to the occurrence of risk event caused by competition among companies.

- Innovation Risk: Innovation risk defined as any risk driven by the low capability of the company in innovating their business process and practice.

- Environmental Risk: This third risk is a kind of risk affecting the non-compliance to environmental requirement/standard

- Security Risk: Security risk occurred when non-compliance to security standard has occurred.

- Social Risk: Social risk is a kind of risk caused by interaction among humans in the operation of a supply chain.

- Political Risk: Political risk is a kind of risk caused by the influence of politics operating in the operation of the supply chain. It is also called governmental risks.

- Human resource risk: Human resource risk is caused by the incompetence of personnel, intended and unintended behavior of human.

- Cultural Risk: This kind of risk is caused by the bad habits of people, society or communities causing or hindering operability of businesses.

- Collaborative Risk: Collaborative risk is a kind of risk caused by collaborating activity among companies and their tiers.

\section{FRAMEWORK FOR SUPPLY CHAIN SUSTAINABILITY RISK ASSESSMENT INDEX}

Intended to measure the adverse impact of sustainable supply chain risk variables, it is necessary to develop a framework to enable decision makers in planning mitigation strategies Upon identifying the most critical sustainability risk variables, decision makers may determine appropriate risk mitigation strategies. In order to develop a framework for evaluating criticality of the supply chain, the element of risk reprioritization approach should be identified. Referring to the definition of risk as the product of probability occurrence of risk events and severity level of their occurrence [12], this definition is possessing limitation since it does not consider various factors affecting the level of risk. In this paper, the supply chain risk reprioritization consists of probability components, loss components, time components, risk worsening factors, and decision maker risk attitude components. The brief elaboration of those components is described in the followings.

- Probability Components

Probability components in the supply chain risk reprioritization approach are about expressing the occurrence of supply chain risk events as a random variable. In this context, the probability components consist of the probability of risk occurrence level, a likelihood of supply chain risk detection and a probability of recoverability when the risk event occurred. The probability of risk occurrence represents the probability level a 
certain sustainability risk variable will occur. When historical data is available, decision makers can estimate quantitatively the probability level that a particular sustainability risk will occur. Otherwise, the use of judgmental approach by decision maker can be accomplished. A probability of detection scale represents the likelihood that a detection procedure owned by the company will be able to detect the cause of a certain sustainability risk event occurs. In other words, detectability probability represents the capability of the focal company detecting the supply chain triggering factors i.e. the probability of the company to identify the cause of the risk variables. The probability of recovery relates to the possibility of a firm enable to recovery after suffering from the effect of a certain risk variable occurrence.

\section{- Loss component}

Loss component relates to the severe impact of the occurrence of sustainability risk variables. Representing the scale of impact of risk variable occurrence effect, loss component in risk assessment is commonly articulated using an ordinal scale. A numerical scale 0 represents to the impossibility that a certain sustainability risk event will occur, meanwhile numerical scale 1 represents certainty that a certain sustainability will occur.

- $\quad$ Time Components

Time components in the risk assessment consists of the interval time of a particular cause of risk event to occur, the time occurrence of a risk event and the estimated time interval an effect of risk impact will occur. When a risk event occurs, the attribution of its risk incurred by its event occurrence is not only on the severity of the effect will be but also on the estimated time span of risk effect consequences. Emerging as an overlooked factor in appraising the criticality of risk in previous FMEA models, the risk time duration is an additional attribute affecting the magnitude of the risk loss consequences.

- $\quad$ Risk Worsening Factors

Risk worsening factor is defined as any factors affecting the escalation of the severity of the risk event consequences. Following Plues et al., [35] it should be considered in estimating the severity of risks. On condition that not all worsening factors affect equally to sustainability risk factors, the correlation between risk worsening and a particular sustainability risk should be included in estimating the impact of risk worsening factors.

\section{- $\quad$ Risk Attitude of supply chain decision makers}

Risk attitude of decision makers is the factor related to the attitude of decision makers in perceiving the impact of a particular risk based on their psychological perception. Those attitudes consist of risk neutral, risk-averse and risk seeker. Consideration of risk attitude in risk assessment is important since it affects the solution chosen by decision makers in tackling the risk [36]. Our approach in considering the scale of sustainability risk parameters is different from the model of Valinejad and Rahmani [37].

Linking all the above factors for accessing the sustainability risk, a framework for evaluating criticality of sustainability indices is provided in FIGURE 1. 


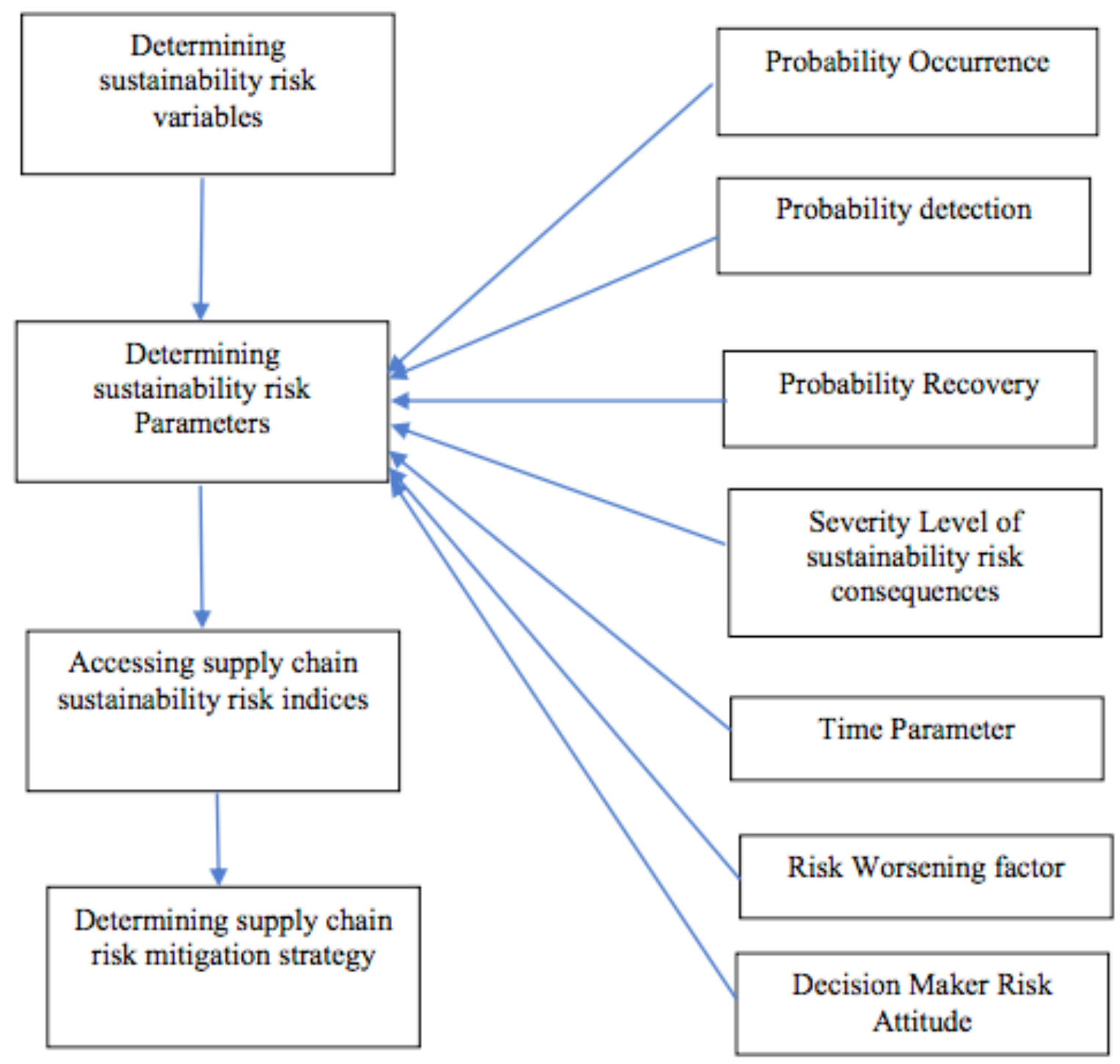

FIGURE 1. A Framework for accessing sustainability risk criticality using new additional risk parameters 
According to FIGURE 1, supply chain sustainability risk assessment consists of three stages, in the first stage involves identification of supply chain sustainability risk indicators and their classification into clusters according to technical, economic, social and cultural dimensions. In the second stage, parameters for risk quantification is determined which consist of probability components, severity components, time aspect, waste worsening factors and risk attitude of decision makers. Those factors become input parameters to quantify the scale of supply chain sustainability risk. In the third stage, determination of the appropriate risk mitigation strategies is proposed.

\section{CONCLUSION}

Driven by changing global business orientation toward practicing sustainable operation in the enterprises' daily operation, identification of sustainability risk factors affecting the operability of the supply chain is important to achieve sustainability. In this paper, differentiation and classification of operational and sustainability risk are proposed and followed by a new framework for sustainability risk assessment. The new framework involves inclusion for new parameters such as time aspect of risk attribution, risk worsening factors, and risk attitude of supply chain decision makers. Attempting to fill the research gap in supply chain risk management field, this initial study can be extended in several paths. In the first path, applying the conceptual model of sustainability into real industrial practice will enable decision makers and academia to obtain insights on the relevance of sustainability risk modes proposed in this paper. In the second path, it is important to investigate the interrelationship and hierarchy of sustainability risk variables for a better understanding of the appropriate risk mitigation strategy.

\section{ACKNOWLEDGMENT}

The authors extremely thankful to the Royal Academy of Engineering (RAE) -UK for financially supporting this study (Funding Reference: IAPP-1/10003).

\section{REFERENCES}

1. H. Karadag, J.Small Bus and Ent.Dev., 4, 22-31 (2016).

2. S. Gao, S. Oreal and J. Zhang, J. Risk and Con. Man.,3, 31-42 (2017).

3. E.Elahi, Foresight, 15,117-131(2012).

4. C. Ellengard, Supp. Ch. Man., An Int. J., 13, 25-34 (2008).

5. M. N. Faisal, Int.J. Inf.Tech. and Supp. Cha. Man., 4, 64-83, (2013).

6. A. Qazi and B. Gaudensi, Int. J. Supp.Ch. and Op.Res.,2, 12-50 (2016).

7. S. Kot, Sustainability, 10, 1-19 (2018).

8. B.P. Sunjka and M.S. Chick, Procd. Int.Conf. CIE, (2012), p. 1-15.

9. G. Berisha and J.S. Pulla, J. Bus., Adm., Law and Soc.Sci., 1,17-28 (2015).

10. A. Rostami, J. Sommerfield, I.L.W.C. Lee, Eng. Const. and Arch.Eng., 22, 91-107 (2015).

11. J.Eduardsen and S.Marinova, Int.J. Expt. Mkt., 1, 4-26, 2016.

12. H. Stamatis (1995), ASQ Press, Milwaukee.

13. W. Ho, T. Zheng, H. Yildiz and S. Talluri, Int.J.Prod. Res., 53,5031-5069 (2015).

14. Y. Fan and M. Stevenson, Int.J. Phy. Dis. And Log. Man., 48,205-230 (2018).

15. Wang, F. Jie and A. Abbareshi, Procd. Op. and Sup.Cha.Int. Conf. Edited by Pujawan et al., (2014), pp.1-9.

16. S. Rasheed,C.F. Wang and S.Memon, I.Ind.Eng and Man., 8,738-762 (2015).

17. S. Nalussamy and P. Ambedkar, Adv.Eng. For.,14,63-71 (2016).

18. S Gupta, M. Goh, R.D.Souza, F.Meng, and M.Garg., Int. J. Inf. Sys. And Supp.Ch. Man., 7, 1-23 (2014).

19. A. Shafiq, P.F.Johnson, R.D.Klaasen and A.Awayshah, Int.J.Opr.Prod. Man., 37, 1386-1407 (2017).

20. N. Paulosis, L.Luong and K. Abhary, WIT Trans. Inf. And Comm. Tech., 39, 121-128 (2008).

21. J. Schultze and S. Halsted, Procd. 21-st Int. Conf. Eng. Des. (2017), pp.

22. F.Lemke and H.L. Petersen, Supp.Cha.Man., An Int. J., 18, 413-429 (2015). 
23. Aon (2011) Global Risk Management Survey 2015, available at:

http://www.aon.com/2015GlobalRisk/attachments/2015-Global-Risk-Management-Report-230415.pdf.

24. N. Mansor, S.N.Yahya,and K. Okazaki, IJRRAS, 27, 18-25 (2016).

25. M. Gianakis and T. Papadopoulos, Int. J. Prod. Econ., 171,455-470 (2016).

26. H. Davarzani,R.Z. Farahani, H.Rahmandad, Int.J.Opr. Prod. Man., 35,1567-1529 (2015).

27. R. Rathore, J.J. Thakkar and J.K. Jha, Int.J. Log. Man., 28, 1272-1293 (2017).

28. M. Meyer, G.Roudt and M. Robbins, S. Af. J. of H. R. Man., 9, 1-12 (2011).

29. Y. Zuo and W.C. Hu,Int.J. Inf.Sys. and Supp.Ch. Man.,2, 19-34 (2009).

30. R. A. Hadiguna, Int. J. Log and Glob., 4, 35-54 (2017).

31. B.J. Galli,Int.J. Risk and Cont. Man., 7,1-39 (2018).

32. K.M.K. Ragunath and S.L.T. Devi,Int.J.Inf.Sys. and Supp.Ch.Man., 11, 87-103 (2018).

33. D. Friday, S. Ryan, R.Shridharan and D. Collins, Int.J. Phy.Dis.Log.Man, 48, (2018).

34. M.S. Monteira, F.L. Viana and J.M.S. Filho, Corp. Gov., In press (2018).

35. D.N. Plues,A. Groso and T.Meyer Chem.Eng. Transc, 21,307-3012 (2013).

36. I. Heckman, T. Comer and S. Nickel, Omega, 52, 119-132 (2014).

37. F. Valinejad and D.Rahmani, J.Cleaner Prod., 203, 53-67 (2018). 